\title{
Dielectric and Spontaneous Polarization Studies of a Ferroelectric Chiral Smectic Liquid Crystal
}

\author{
Abraham K. George $^{1 *}$, C. Carboni ${ }^{1}$, S. H. Al-Harthi ${ }^{1}$, W. M. Zoghaib ${ }^{2}$, D. M. Potukuchi ${ }^{3}$ \\ ${ }^{1}$ Department of Physics, Sultan Qaboos University, Muscat, Oman; ${ }^{2}$ Department of Chemistry, Sultan Qaboos University, Muscat, \\ Oman; ${ }^{3}$ Department of Physics, University College of Engineering, Jawaharlal Nehru Technological University, Kakinada, India. \\ Email: *akgeorge@squ.edu.om
}

Received November $10^{\text {th }}, 2011$; revised December $16^{\text {th }}, 2011$; accepted December $28^{\text {th }}, 2011$

\begin{abstract}
Dielectric relaxation and spontaneous polarization studies have been carried out in a low molar mass organosiloxane liquid crystal, TSi10PPBN4 which displays a wide temperature range of de Vries Smectic A* and chiral Smectic C phases. The temperature variation of the permittivity and dielectric loss studied are found to have anomalous variations in the vicinity of the phase transitions. The frequency variation of permittivity and dielectric loss are also studied in the de Vries Smectic $A^{*}$ and Smectic $C^{*}$ phases. The relaxation frequency, $f_{R}$ is found to decrease with decreasing temperature. Temperature variation of $f_{R}$ is found to follow an Arrhenius shift from which the activation energy is estimated. The thermal variation of the spontaneous polarization, $P_{s}$ is measured using the polarization reversal technique in the Smectic $C^{*}$ phase. $P_{s}$ increases initially, reaches a peak value and then drops drastically in the vicinity of the $\mathrm{SmC}^{*}-\mathrm{SmA}_{\text {de Vries }}^{*}$ phase transition.
\end{abstract}

Keywords: Smectic $\mathrm{C}^{*}$ and Smectic $\mathrm{A}_{\text {de vries }}^{*}$ Phases; Dielectric Permittivity; Dielectric Relaxation; Activation Energy; Spontaneous Polarization

\section{Introduction}

Amongst the wide variety of experimental methods used to study the molecular dynamics of liquid crystals (LCs), the low frequency dielectric spectroscopy assumes special importance due to the utility of the relevant data in electro-optic devices. These LC devices are known to operate in the frequency region of a few $\mathrm{Hz}$ to several $\mathrm{MHz}$ that involves different modes of their molecular motions. Since these molecular motions involve both collective and non-collective types, dielectric spectroscopy is evolving as a powerful tool for the study of performance of LCs in devices. Measurement of the LC dielectric response enables one to determine the relaxation time, which is an important dynamical quantity of the molecules.

The discovery of ferroelectric liquid crystals (FLCs) and the advent of $C^{*}$-based surface stabilized geometry [1] with sub microsecond switching speed has further opened up a diverging field of applications in fast LC electro-optic switching devices such as the optical modulators, spatial light modulators, high definition displays, etc. The key point in their application is exclusively based on our understanding of their molecular motion in response to an applied field. However, as they

\footnotetext{
${ }^{*}$ Corresponding author.
}

belong to the class of thermotropic LC phases, it is equally important to study the influence of temperature on the underlying orientation order (like the $\mathrm{C}^{*}$ tilt angle and the spontaneous polarization $P_{S}$ ) in the liquid crystalline phase of devise interest. The tilt angle in the Smectic $\mathrm{C}^{*}\left(\mathrm{SmC}^{*}\right)$ phase with respect to the smectic layer normal is considered as the primary order parameter, while the spontaneous polarization is considered as the secondary order parameter. The data obtained from dielectric and optical studies provide a further directive for the preferred design and synthesis of FLC material with an optimized performance.

The flexible terminal tail groups of most of the FLCs are reported to possess prevalently an alkyl or alkoxy chains (as methyline units consist of hydrogen atoms) at the flexible ends. Replacement of the alkyl chains with either fluorocarbons or siloxy units is also reported [2-4] for the optimization of their performance in devices. Replacement of alkyl chains partially with dimethylsyloxane groups is found $[5,6]$ to enhance the thermal stability of the underlying smectic phase and to lower the transition temperatures towards ambient temperature. Dimethylsiloxane groups are bulky and exhibit comparatively irregular configurations than the alkyl (or alkoxy) end chain counterparts. As a result, a reduced coupling be- 
tween the neighboring mesogenic moieties (within a layer or over the layers) is anticipated. Thus, the reduced coupling between the neighboring mesogens favors the formation [7] of a de Vries type Smectic A* $\left(\mathrm{SmA}_{\text {de Vries }}^{*}\right)$ phase. In these de Vries phase materials, the molecules are tilted with respect to the layer normal. The tilt in de Vries phases does not possess [7] long-range correlation. Hence, the averaged optic axis of the material is configured normal to the smectic layer. As the chiral $\mathrm{SmC}^{*}$ phase is a layered structured phase made up of chiral molecules, it is attributed to contain a modulated structure of the director (i.e., while going from one layer to the neighboring layer along the layer normal). As a result, a striped microscopic texture appears revealing the presence of a super structure (a helix) in the sample. This helix need to be deformed (unwound by the application of external fields) as to harness the polarization to design a switching in a device. Due to the presence of chiral molecules, $\mathrm{SmC}^{*}$ phase exhibits ferroelectric properties.

In this paper, we report the results of the dielectric and spontaneous polarization studies carried out in a monomeric low molar mass organosiloxane liquid crystal material, viz., TSi10PBN4 which exhibits wide temperature ranges for $\mathrm{SmA}^{*}{ }_{\mathrm{de}}$ Vries and $\mathrm{SmC}^{*}$ phases. After the general introduction, details of the experimental techniques used for the present study is presented in the second section. The results of the dielectric relaxation studies and the spontaneous polarization are presented in the third section. The results are discussed in the next section followed by summary and conclusion.

\section{Experimental}

\subsection{Material}

TSi10PBN4 was synthesized following the earlier reported [8] procedure. The chemical formula of the compound is given in Figure 1.

An Olympus (BH-2) polarizing microscope in conjunction with a Linkam (TMS 94) heating stage was used for the microscopic textural observations. The cooling and heating rates followed during the microscopic studies are $0.5^{\circ} \mathrm{C}$ per minute.

Microscopic textural observations confirmed the following phase sequence:

Crystal $\stackrel{-34^{\circ} \mathrm{C}}{\longrightarrow} \mathrm{SmC}^{*} \stackrel{27^{\circ} \mathrm{C}}{\longleftrightarrow} \mathrm{SmA}_{\text {de Vries }}^{*} \stackrel{100^{\circ} \mathrm{C}}{\longleftrightarrow}$ Isotropic
The compound is found to exhibit practically invariant phase transition temperatures as well as identical phase sequence over repeated cycles of heating and cooling. These observations reflect upon the stability and purity of the sample.

\subsection{Dielectric and Spontaneous Polarization Measurements}

Commercially available (Device Tech Inc., USA) cells made up of a pair of transparent polyamide buffed glass plates of $10 \mu \mathrm{m}$ separation were used for the dielectric studies. The LC was introduced into the cell by capillary action in its high temperature isotropic state. During the filling, the cell was observed through a polarizing microscope, with crossed polarizer configuration to ensure the homogeneous alignment of the sample. The cell was placed in a special oven, and the temperature was stabilized to $\pm 0.1^{\circ} \mathrm{C}$ with a Linkam TMS 94 temperature controller. The experimental studies were carried out by heating the sample to an initial temperature of around $5^{\circ} \mathrm{C}$ higher than its clearing temperature in to isotropic state, followed by its slow cooling (about $0.5^{\circ} \mathrm{C}$ per minute) to the temperature of interest in the liquid crystalline phases.

The dielectric permittivity $\varepsilon^{\prime}(\omega)$ and the loss factor $\tan \Delta(\omega)$ measurements were carried out in the frequencies range of $5 \mathrm{~Hz}$ to $13 \mathrm{MHz}$ using a Hewlett Packard Impedance Analyzer (HP-LF 4192A) in conjunction with an HP-16047C Test Fixture Kit. The empty cell was calibrated with standard organic solvents like Benzene and the leads permittivity $\varepsilon_{\text {leads }}^{\prime}$ was determined at room temperature. $\varepsilon_{\text {leads }}^{\prime}$ was found to be invariant with temperature within the range of the present investigation.

The spontaneous polarization was measured using a triangular wave $\left(60 \mathrm{~V}_{\mathrm{p}-\mathrm{p}}\right)$ of $12 \mathrm{~Hz}$ frequency by the polarization reversal technique [6].

\section{Results and Discussion}

\subsection{Dielectric Permittivity}

The temperature variation of the dielectric permittivity and the dielectric loss are studied using $1 \mathrm{~V}$ oscillating level at $100 \mathrm{kHz}$ frequency as a function of temperature. The temperature variation of permittivity is not associated with any abrupt change in the vicinity of any of the phase transitions, as observed in some other LCs $[9,10]$.<smiles>CCCCCOc1ccc(-c2ccc(C(=O)Oc3ccc(OC(C)C(=O)OCC)c([N+](=O)[O-])c3)cc2)cc1</smiles>

Figure 1. Chemical formula of TSi10PBN4. 
We have therefore estimated the temperature derivative of permittivity and the results are presented in Figure 2. The temperatures at which abrupt variations occur are considered to be the transition temperature. The $\mathrm{SmA}_{\text {de Vries }}^{*}$ to isotropic transition at $100^{\circ} \mathrm{C}$ and the $\mathrm{SmC}^{*}$ to $\mathrm{SmA}_{\text {de Vries }}^{*}$ at $27^{\circ} \mathrm{C}$ observed form the low frequency dielectric measurements agree with microscopic textural observations given in experimental section.

\subsection{Dielectric Relaxation Studies}

The observed frequency variation (5 to $13 \mathrm{MHz}$ ) of permittivity, $\varepsilon^{\prime}(\omega)$ and the loss factor $\tan \Delta(\omega)$ from the impedance analyzer (at a given temperature $T$ in the phase of interest) are used to estimate the dielectric loss, $\varepsilon^{\prime \prime}(\omega)$. The dielectric loss $\varepsilon^{\prime \prime}(\omega)$ is estimated by multiplying $\tan \Delta(\omega)$ with $\varepsilon^{\prime}(\omega)$. An a.c. sinusoidal wave of $1 \mathrm{~V}_{\mathrm{p}-\mathrm{p}}$ with variable frequency is used for the studies. The dielectric relaxation studies are carried out in the $\mathrm{SmA}^{*}{ }_{\mathrm{de} \text { Vries }}$ and $\mathrm{SmC}^{*}$ phases at different specified temperatures. The dielectric loss spectra (i.e., the frequency variation of the loss) studied at different temperatures in the $\mathrm{SmC}^{*}$ and $\mathrm{SmA}_{\text {de Vries }}^{*}$ phases are presented in Figures 3 and 4 , respectively.

It has been observed that the dielectric loss exhibits a peak in the low frequency region, in both $\mathrm{SmA}_{\text {de Vries }}^{*}$ and $\mathrm{SmC}^{*}$ phases. The frequency at which the dielectric loss attains maximum value is identified as the relaxation frequency $f_{R}$.

The relaxations in $\mathrm{SmC}^{*}$ phase are reported to be associated with [11-13] with two different types, viz., one around few hundred $\mathrm{Hz}$ and the other in the $\mathrm{MHz}$ region. The former is reported to correspond to the Goldstone mode relaxation, which is related to the reorientation of the molecular transverse moment to the field. The latter $\mathrm{MHz}$ relaxation (high frequency) corresponds to the soft mode relaxation process, which is related to the reorientation of the longitudinal molecular dipole moment to the

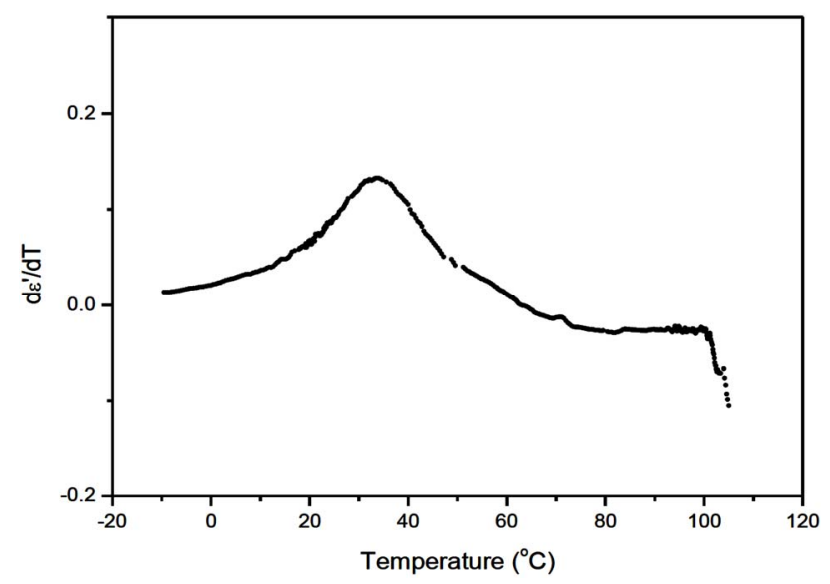

Figure 2. Temperature variation of the differential permittivity.

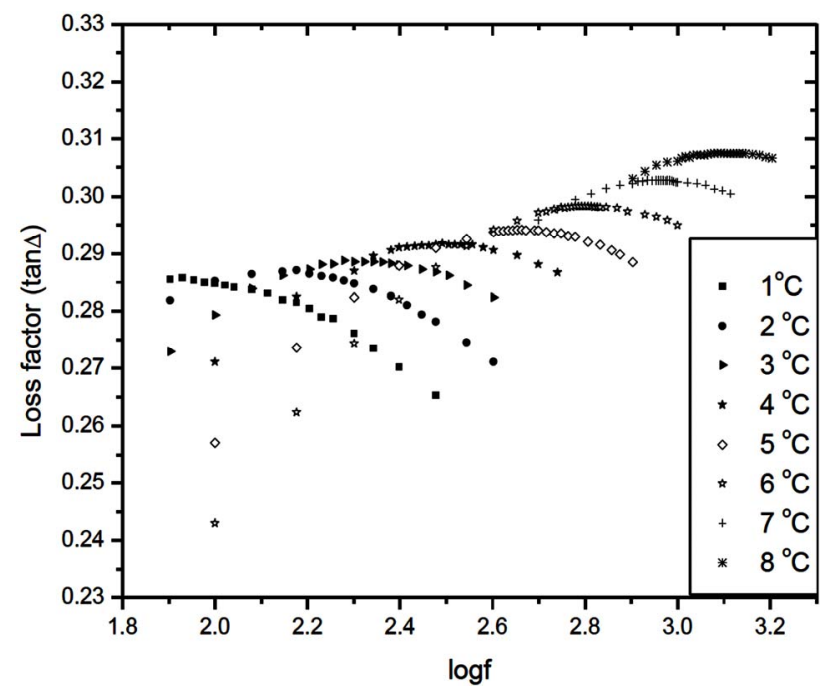

Figure 3. Frequency variation of the loss factor $\tan \Delta$ in the SmC ${ }^{*}$ phase at different temperatures.

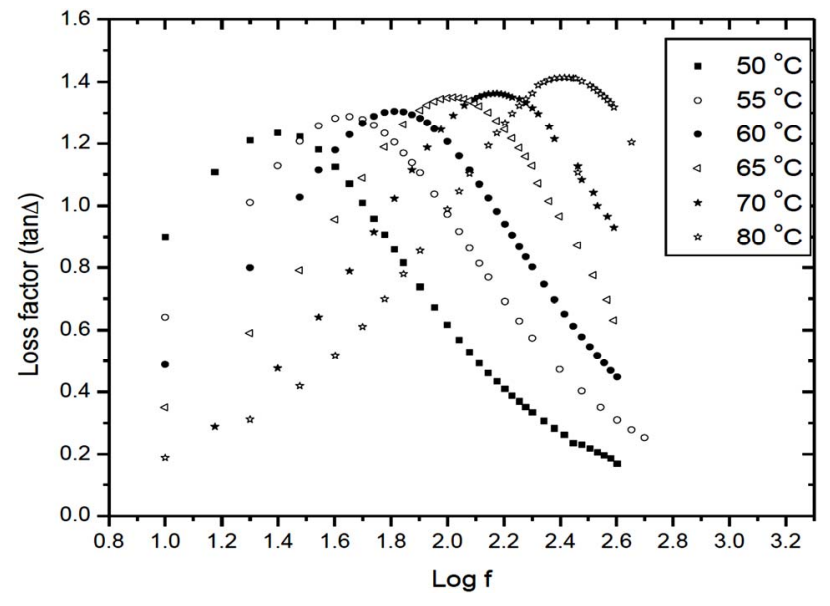

Figure 4. Frequency variation of the loss factor $\tan \Delta$ in the $\mathrm{SmA}^{*}{ }_{\text {de Vries }}$ phase at different temperatures.

field. It may be noticed that the present studies are not accompanied with any soft mode relaxations. The present case of absence of soft mode relaxation in any of the smectic phases is argued as due to the de Vries phase structure, where the long-range order conspicuously is lacking, while tilt is a local order.

It is also observed that the relaxation frequency $f_{R}$ shifts to the lower frequency side with the decreasing temperature in both of the $\mathrm{SmC}^{*}$ and $\mathrm{SmA}^{*}$ de Vries phases (Figure 5).

\subsection{Spontaneous Polarization}

The temperature variation of spontaneous polarization $\left(P_{\mathrm{s}}\right)$ in the $\mathrm{SmC}^{*}$ phase was obtained [6] from the area under the polarization-reversal current peak when a triangular wave is applied to the specimen. The temperature variation of $P_{\mathrm{s}}$ reflecting the secondary order parameter 
is presented in Figure 5. It has been observed that $P_{\mathrm{s}}$ increases initially, reaches a peak value and then drops drastically in the vicinity of the $\mathrm{SmC}^{*}-\mathrm{SmA}_{\text {de }}^{*}$ Vries phase transition. The maximum value $P_{\mathrm{s}}$ is found be as high as over $100 \mathrm{nC} / \mathrm{cm}^{2}$. This high value of $P_{s}$ is comparable [3] to the values measured in other organosiloxane liquid crystals and is attributed to the presence of organosiloxane end chain.

\section{Discussion}

The observed temperature variation of the relaxation frequency is analyzed through the corresponding Arrhenius plots [10,14] in Figures 6 and 7. The estimated activation energy is found to be $6.37 \pm 0.20 \mathrm{eV}$ in the $\mathrm{SmC}^{*}$ phase and $1.90 \pm 0.10 \mathrm{eV}$ in the $\mathrm{SmA}^{*}{ }_{\text {de Vries }}$ phases. This activation energy is found to be on the higher side of the reported $[9,15]$ values. The relatively higher value of the activation energy is suggestive of the higher potential barrier witnessed by the molecular dipole moment to orient to the field characteristic of organosiloxane end chains promoting greater disorder on the LC molecular frame.

In chiral de Vries Smectic-A materials [15], the alignment of the optic axis can be controlled with an electric field $[7,16]$. The mechanism responsible for the tilting of the optic axis is different from that of the normal Electroclinic Effect observed in chiral Smectic A materials. In the de Vries Smectic A* materials, the molecules are tilted with respect to the layer normal, but there is no long-range correlation between the directions of the tilt. The orientation of the long axis of the molecules is uniformly distributed on a cone with its cone angle equal to twice the molecular tilt. The optic axis of the material, which is considered to be the average molecular orientation, is therefore normal to the layers. Hence, the symmetry of the phase is that of an ordinary Smectic A phase. However, as a local order (within a small volume) in the range of the correlation length prevails between the two physically equivalent directions of the molecular tilt.

As the molecule possesses a chiral centre and organized in tilted configuration on a short-range scale, a polarization results in the plane of the layer and normal to the local tilt direction. Due to the uniform distribution of the local tilt, there is no net macroscopic polarization. The application of an electric field along the layer planes of the layers thus favors the molecular re-orientation, so that the induced polarization would be to align to the field. Therefore, the field produces a biased distribution of molecular tilt with respect to the average optic axis. This variation in tilt produces considerable variation in birefringence (with the applied field) as a characteristic feature of the $\mathrm{SmA}_{\text {de Vries }}^{*}$ phase. The tilt increases with increasing field until all the molecules have the same direction of tilt (so that the polarization is parallel to the

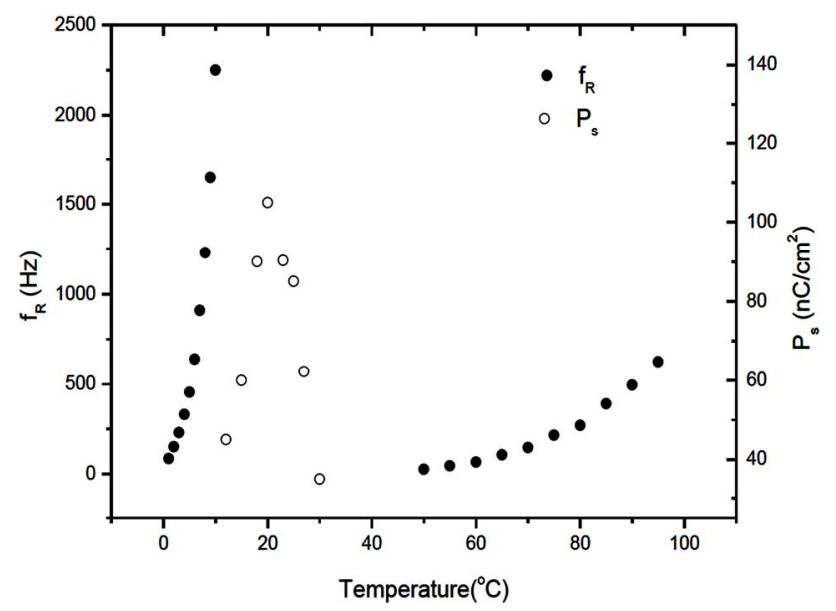

Figure 5. Temperature variation of relaxation frequency $f_{R}$ in the $\mathrm{Sm} \mathrm{A}_{\text {de Vries }}^{*}$ and the $S m \mathrm{C}^{*}$ phases and the spontaneous polarization $\left(P_{s}\right)$ in the $S m C^{*}$ phase.

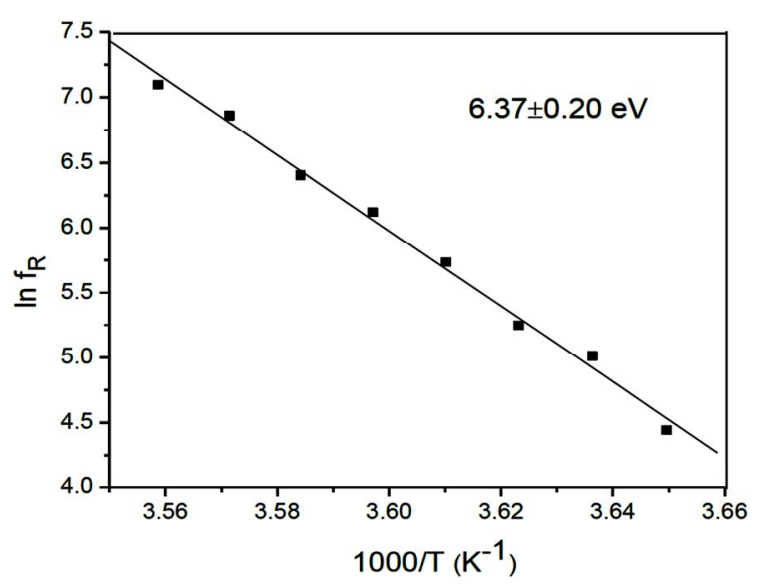

Figure 6. Reduced temperature plot (Arrhenius) for the low frequency mode relaxation in the $\mathrm{SmC}^{*}$ phase.

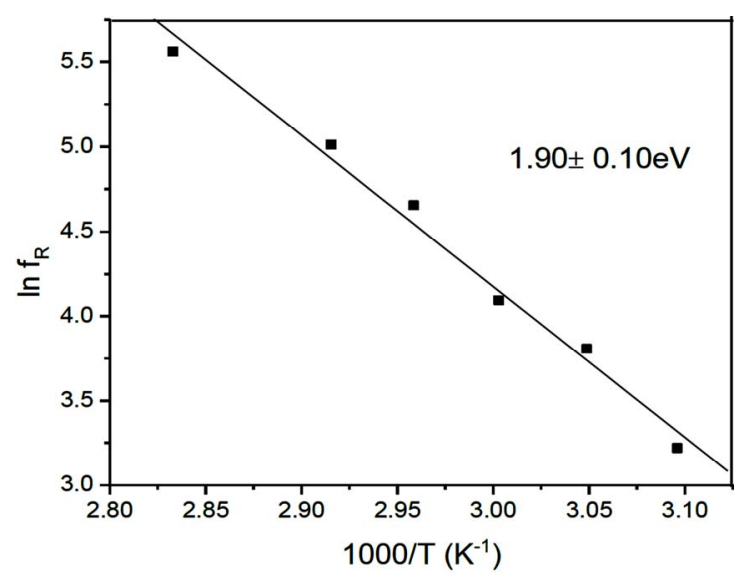

Figure 7. Reduced temperature plot (Arrhenius) drawn for the low frequency mode relaxation in the $\mathrm{SmA}^{*}{ }_{\text {de Vries }}$ phase.

field). The tilt of the optic axis increases with the field and saturates at an angle equal to the molecular tilt. A defined restraint over this de Vries phase is that such a 
variation in tilt should not be accompanied with the expected variation in the smectic layer spacing. However, this leads to a large induced tilt angle, possibly as a preferred aspect in devices. Furthermore, the observed steep increase of $P_{S}$ in $\mathrm{SmA}^{*}$ de Vries phase for a temperature $>$ $27^{\circ} \mathrm{C}$ is found to agree with the detailed mechanism for its realization.

\section{Summary and Conclusions}

From the present study it may be concluded that:

1) Thermal microscopic studies could reveal the occurrence of $\mathrm{SmC}^{*}$ and $\mathrm{SmA}^{*}{ }_{\text {de Vries }}$ phases in the material studied.

2) Dielectric parameter studied as a function of temperature can be used to determine the phase transitions temperature in LCs. Although, a phase transition is not accompanied with an abrupt change in dielectric parameter, the anomaly associated with its temperature derivative could be a tool to identify the phase transitions involving LC phases.

3) The frequency variation of permittivity $\varepsilon^{\prime}(\omega)$ and the loss factor Tan $\Delta$ studied at different temperatures in both of the the $\mathrm{SmC}^{*}$ and $\mathrm{SmA}_{\text {de Vries }}{ }^{*}$ phases is useful to determine the relaxation frequency $f_{R}$. The temperature variation in $f_{R}$ (shifting to the lower frequency side) in both of the $\mathrm{SmC}^{*}$ and $\mathrm{SmA}_{\text {de Vries }}^{*}$ phases suggests the involvement of activation energy reflecting the strength of the potential barrier. Activation energy is found to be higher in de Vries phases than in ordinary LC phases.

4) The spontaneous polarization increases initially, which attains a peak value, while it drops drastically as the $\mathrm{SmA}_{\text {de Vries }}^{*}$ phase sets in.

\section{REFERENCES}

[1] G. R. Luckhurst and C. A. Veracini, "The Molecular Dynamics of Liquid Crystals," Kluwer Academic Publishers, Dordrecht, 1994.

[2] J. Naciri, J. Ruth, G. Crawford, R. Shashidar and B. R. Ratna, "Novel Ferroelectric and Electroclinic Organosiloxane Liquid Crystals," Chemistry Materials, Vol. 7, No. 7, 1995, pp. 1397-1402. doi:10.1021/cm00055a019

[3] W. K. Robinson, P. S. Kloess, S. P. Perkins, C. Carboni and H. J. Coles, "Ferroelectric and Antiferroelectric Low Molar Mass Organosiloxane Liquid Crystals," Liquid Crystals, Vol. 25, No. 3, 1998, pp. 301-307. doi:10.1080/026782998206092

[4] W. K. Robinson, P. Lehman and H. J. Coles, "Bi-Mesogenic Fluorinated Organosiloxanes: An Electro-Optic Study Revealing Unusual Phase Behavior," Molecular Crystals and Liquid Crystals, Vol. 328, No. 1, 1999, pp. 229-236. doi:10.1080/10587259908026063

[5] A. Kaeding and P. Zugenmaier, "Investigations of a Homologous Series of Chiral Siloxane Based Dimesogenic
Compounds," Liquid Crystals, Vol. 25, No. 4, 1998, pp. 449-457. doi:10.1080/026782998205958

[6] K. Miyasato, S. Abe, H. Takezoe, A. Fukuda and E. Kuze, "Direct Method with Triangular Waves for Measuring Spontaneous Polarization in Ferroelectric Liquid Crystals," Japanese Journal of Applied Physics, Vol. 22, 1983, pp. L661-L663. doi:10.1143/JJAP.22.L661

[7] J. V. Selinger, P. J. Collins and R. Shashidar, "Field Dependent Tilt and Birefringence of Electroclinic Liquid Crystals," Physics Review E, Vol. 64, No. 6, 2001, pp. 1-9.

[8] J. Naciri, C. Carboni and A. K. George, "Low Transition Temperature Organosiloxane Liquid Crystals Displaying a de Vries Smectic A Phase," Liquid Crystals, Vol. 30, No. 2, 2003, pp. 219-225. doi:10.1080/0267829021000060250

[9] D. M. Potukuchi, A. K. George, C. Carboni, S. H. Al-Harthi and J. Naciri, "Low Frequency Dielectric Relaxation, Spontaneous Polarization, Optical Tilt Angle and Response Time Investigation in a Fluorinated Ferroelectric Liquid Crystal," Ferroelectrics, Vol. 300, No. 1, 2004, pp. 79-93. doi:10.1080/00150190490442245

[10] A. K. George, M. Al-Hinai, D. M. Potukuchi, S. H. Al-Harthi and C. Carboni, "Study of Dipole Dynamics and Pre-transitional Effects at Isotropic to Nematic Phase Transition by Low-Frequency Dielectric Relaxation Measurements," Phase Transitions, Vol. 76, No. 12, 2003, pp. 1037-1045. doi:10.1080/0141159031000114865

[11] M. Wojciechowski, L. A. Gromice and G. W. Bąk, "Dielectric Relaxations in Liquid Crystalline 4-(1-Methylheptlocabonyl)phenyl-4'-( $\omega$-perfluoroacetoxypropoxy)biphen yl-4-carboxylate," Journal of Molecular Liquids, Vol. 86, No. 1-3, 2000, pp. 269-272. doi:10.1016/S0167-7322(99)00148-8

[12] B. Žekš and R. Blinc, "Dielectric Properties of Smectic C" Liquid Crystals," Molecular Crystals and Liquid Crystals, Vol. 220, No. 1, 1992, pp. 63-65. doi:10.1080/10587259208033429

[13] P. G. Castello, Y. P. Kalmykov and J. K. Vij, "High Frequency Dielectric Behavior of a Ferroelectric Liquid Crystal near the Smectic C ${ }^{*}$-Smectic A Phase Transition," Physical Review A, Vol. 46, No. 8, 1992, pp. 4852-4858.

[14] A. K. George, M. Al-Hinai, D. M. Potukuchi, C. Carboni, S. H. Al-Harthi and J. Naciri, "Dielectric Response in the Smectic A and Smectic C ${ }^{*}$ Phases of a Ferroelectric Liquid Crystal, 12CN5(R*)," Molecular Crystals and Liquid Crystals, Vol. 409, No. 1, 2004, pp. 343-353. doi:10.1080/15421400490431714

[15] A. de Vries, "Experimental Evidence Concerning Two Different Kinds of Smectic C to Smectic A Transitions," Molecular Crystals and Liquid Crystals, Vol. 41, No. 2, 1977, pp. 27-31. doi:10.1080/01406567708071949

[16] C. Carboni, R. Mehdi, A. K. George and S. H. Al-Harthi, "Electroclinic Effect in Low Molar Mass Organosiloxane Liquid Crystal," Molecular Crystals and Liquid Crystals, Vol. 410, No. 1, 2004, pp. 61-69. doi:10.1080/15421400490434937 